\title{
Mosaic Masonries to Interpret Diagnostic Test Results
}

\author{
Ramalingam Shanmugam \\ School of Health Administration, Texas State University, San Marcos, TX 78666, USA
}

Received 2012-10-20, Revised 2013-01-05; Accepted 2013-02-12

\begin{abstract}
Currently, assessing superiority of a diagnostic test requires cumbersome calculations and confusing interpretations because of nonlinear Receiver Operating Characteristic Curve (ROC). To remedy these difficulties, this article prepares, provides an easier alternate geometric approach. That is, a simple but more effective visual approach with two complementary mosaic masonries are constructed with their properties, superimposed and utilized. First masonry is conditional on disease status. The second masonry is conditional on test outcomes. By superimposing both masonries and visualizing their overlap territory, our approach utilizes an angle, $\theta$ to assess superiority of a diagnostic test. A hypothesis testing procedure is also devised to compute the significance level of an estimated angle. The statistical power to accept a true angle is developed in the procedure. In the end, the concepts, properties and advantages of mosaic masonries are illustrated using medical and public health data sets.
\end{abstract}

Keywords: Sensitivity, Specificity, Prevalence, Geometry, Youden Index, ROC Curve, Positive and Predictive Values

\section{INTRODUCTION}

\subsection{Motivation for Visuals}

Quite often, a prelude to select a suitable medical treatment, a physician or surgeon demands the patient to undergo a diagnostic test. For examples, the urine, blood, tissue, EKG, X-rays, sonogram and other laboratory test outcomes are utilized to select an appropriate treatment for a patient. The diagnostic test results provide a clue or confirmatory evidence about an illness. Not everyone with a positive result $(+)$ is necessarily inflicted with an illness. This idea is called Positive Predictive Value (PPV). Likewise, not everyone with a negative result (-) is necessarily healthy with immunity to an illness. This idea is called Negative Nredictive Value (NPV).

To be specific, note that Equation 1 and 2:

$$
\operatorname{PPV}=\operatorname{Pr}(\mathrm{D} \mid+)
$$

And:

$$
\mathrm{NPV}=\operatorname{Pr}(\overline{\mathrm{D}} \mid-)
$$

where, the notations $\mathrm{D}$ and $\overline{\mathrm{D}}$ denote an event of illness or healthy state respectively. To further understand, suppose that $p_{+}$and $p_{-}$denote respectively the proportion of participants with positive and negative result in a diagnostic test. Then, $\mathrm{H}_{\mathrm{D}+}=\mathrm{p}_{+} \mathrm{PPV}$ and $\mathrm{H}_{\overline{\mathrm{D}}-}=\mathrm{p}-\mathrm{NPV}$ portray harmony in the sets $(+, \mathrm{D})$ and $(-, \overline{\mathrm{D}})$ respectively.

On the contrary, $\mathrm{H}_{\overline{\mathrm{D}}+}=(1-\pi)\left(1-\mathrm{S}_{\mathrm{p}}\right) \quad$ and $\mathrm{H}_{\mathrm{D}-}=\pi\left(1-\mathrm{S}_{\mathrm{e}}\right)$ signify discard in the sets $(+, \overline{\mathrm{D}})$ and $(-, \mathrm{D})$ respectively. The harmonies and discards are the basis to formulate mosaic masonry later in the article.

Alternate to PPV and NPV are sensitivity $\left(\mathrm{S}_{\mathrm{e}}\right)$ and specificity $\left(S_{p}\right)$. In probability terminologies, they are Equation 3 and 4:

$\mathrm{S}_{\mathrm{e}}=\operatorname{Pr}(+\mid \mathrm{D})$

And:

$\mathrm{S}_{\mathrm{p}}=\operatorname{Pr}(-\mid \overline{\mathrm{D}})$ 
Suppose that the proportion of participants with an illness is $\pi=\operatorname{Pr}(D)$ and it is referred as prevalence of the illness. The $S_{e}$ and $S_{p}$ are recognized as True Positive (TP) and True Negative (TN) in the literature. Their complements $1-\mathrm{S}_{\mathrm{e}}$ and $1-\mathrm{S}_{\mathrm{p}}$ are False Positive (FP) and False Negative (FN). However, $\mathrm{H}_{+\mathrm{D}}=\pi \mathrm{S}_{\mathrm{e}}$ and $\mathrm{H}_{-\overline{\mathrm{D}}}=(1-\pi) \mathrm{S}_{\mathrm{p}}$ denote alternate harmonies in the sets $(+$, D) and $(-, \bar{D})$. On the contrary, $H_{+\bar{D}}=(1-\pi)\left(1-S_{p}\right)$ and $\mathrm{H}_{-\mathrm{D}}=(1-\pi) \mathrm{S}_{\mathrm{e}}$ signify alternate discards respectively in the sets $(+, \overline{\mathrm{D}})$ and $(-, \mathrm{D})$. These harmonies and discards are complementary basis to formulate an alternate mosaic masonry later in the article.

The well-known Youden index $I=S_{e}+S_{p}-1$ plays a crucial role to identify a superior diagnostic test. The index, I integrates only sensitivity and specificity and hence, it is a conditional measure of superiority in the known presence or absence of a disease (Youden, 1950). In this sense, the Youden index is a restrictive measure to describe the superiority of a diagnostic test. Larger value of the index, I means more superior diagnostic test only in a restrictive scenario. The value of the index, I does not reveal whether sensitivity or specificity is high and it is a weakness of the index, I. High sensitivity with a low specificity or low sensitivity with a high specificity is a possibility even for a given value of the Youden index. A mosaic masonry to be constructed in this article is a better alternate to the disadvantageous Youden Index, I. The mosaic area denotes superiority of a diagnostic test in the same conditional scenario of Youden index but is more a visual. The mosaic tile area is $\mathrm{S}_{\mathrm{e}} \mathrm{S}_{\mathrm{p}}$ (Fig. 1) which is not large unless both $S_{e}$ and $S_{p}$ are large and in this sense, the tile area is better index than the Youden index.

Likewise, in a superior diagnostic test, the sum of PPV and NPV needs to be large. Both PPV and NPV are built in a less known index, $\mathrm{J}=\mathrm{PPV}+\mathrm{NPV}-1$. Shanmugam (2008) for definition and properties of the index, J. This index is also a restrictive measure to indicate superiority of a diagnostic test only in a given scenario of details about test results. Large value of the index, $\mathrm{J}$ means a superior diagnostic test and vice versa. A high value of the index, J does not indicate whether both PPV and NPV are high and it is a weakness of the index, J. High PPV with a low NPV or low PPV with a high NPV is a possibility for a given value of Shanmugam's index, J. Mosaic masonry to be constructed in this article based on PPV and NPV is a better alternate to the restrictive measure $\mathrm{J}$ about the superiority of a diagnostic test. The mosaic tile area, PPV*NPV (Fig. 2) is large only when both PPV and NPV is large and in this sense, the masonry is preferable to the index, J. The masonry tile area is a visual and easy to construct.

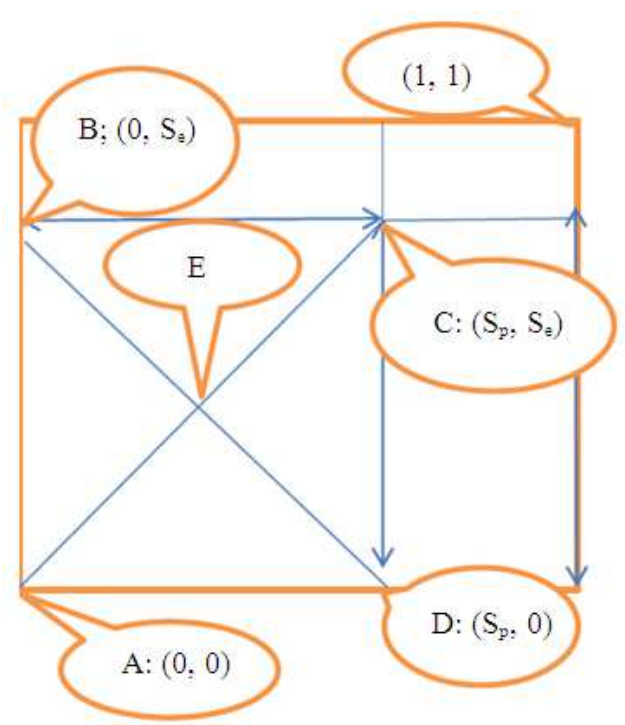

Fig. 1. Mosaic tiles

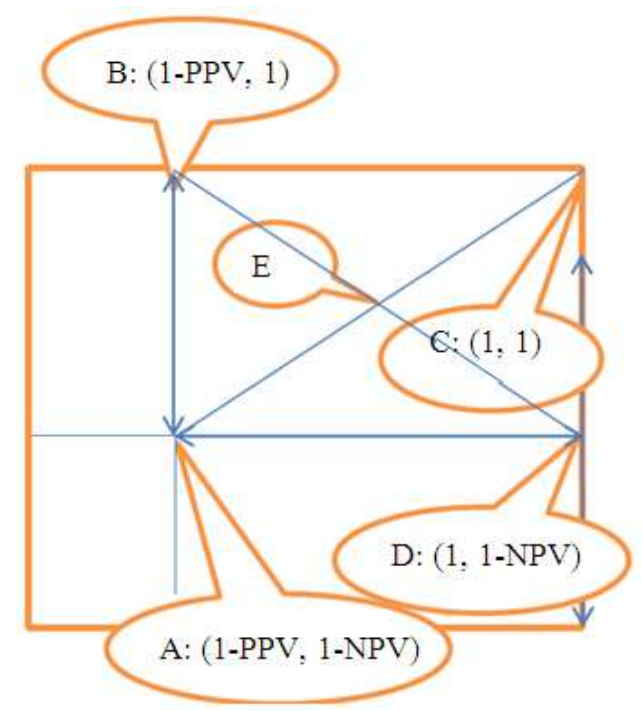

Fig. 2. Complementary mosaic tiles

Both indices, I and $\mathrm{J}$ are linked via a concept which was named double anchors by Shanmugam (2008). He showed that the prevalence, PPV, NPV, $\mathrm{S}_{\mathrm{e}}$ and $\mathrm{S}_{\mathrm{p}}$ are all inter-connected. One anchor weighs $\mathrm{p}^{+} \mathrm{p}_{\mathrm{J}} \mathrm{J}$ and another anchor weighs $\pi(\mathrm{I}-\pi) \mathrm{I}$. When both anchors weigh the same, a diagnostic test is quite balanced. Otherwise, there is an imbalance in a diagnostic test. The imbalance among the anchors is captured by $\delta=\left|\mathrm{p}_{+} \mathrm{p}_{-} \mathrm{J}-\pi(\mathrm{I}-\pi) \mathrm{I}\right|$. Another byproduct of the double anchoring idea is an authentication level. Shanmugam (2008) distinguished a 
circumstantial from compelling evidence using authentication levels.

However the evidences in a diagnostic data analysis turn out, there is a duality between the indices I and J. Both indices are like two sides of a coin. A negative value of I (likewise of J) means an inferior diagnostic test. The positive value of I (likewise of J) refers a superior diagnostic test. Both indices detect a superior diagnostic test when it exists, though the approaches are opposite but conditional. Why not crossbreed both approaches to obtain their bests? This is achieved in this article via superimposed mosaic masonries.

Next, mosaic masonry concepts are formally defined, developed and utilized as an alternate visual approach to ROC. They are illustrated using medical and health data. Lastly in the article, conclusive thoughts are stated.

\subsection{Constructing Mosaic Masonries}

A mosaic masonry connecting sensitivity and specificity can be constructed in a unit square, as in Fig. 1 below, so that the tile areas indicate harmonies $\mathrm{H}_{+\mathrm{D}}=\pi \mathrm{S}_{\mathrm{e}}, \mathrm{H}_{-\overline{\mathrm{D}}}=(1-\pi) \mathrm{S}_{\mathrm{p}}$ and

discards $\mathrm{H}_{+\overline{\mathrm{D}}}=(1-\pi)\left(1-\mathrm{S}_{\mathrm{p}}\right) \mathrm{H}_{-\mathrm{D}}=\pi\left(1-\mathrm{S}_{\mathrm{e}}\right) . \quad$ Various properties of the mosaic in Fig. 1 are explored and catalogued below.

Property 1. The tile area ABCD is $\mathfrak{R}_{1}=\mathrm{S}_{\mathrm{p}}{ }^{*} \mathrm{~S}_{\mathrm{e}}$ which signifies superiority of a diagnostic test. The domain for $\mathfrak{R}_{1}$ is $[0,1]$. Unless both sensitivity and specificity are large, the tile area, $\mathfrak{R}_{1}$ is not large and hence, it is better index than the Youden index, I.

Property 2. The odds for a diagnostic test to be superior is $\operatorname{odd}_{1}=\left(\Re_{1}^{-1}-1\right)^{-1}$. The odds increases when the area increases.

Property 3. The slope of the positive diagonal AC is $\mathrm{m}_{1}=\frac{\mathfrak{R}_{1}}{\mathrm{~S}_{\mathrm{p}}^{2}}$ or equivalently, $\mathrm{m}_{1}=\frac{\mathrm{S}_{\mathrm{e}}^{2}}{\mathfrak{R}_{1}}$ which is just minus of the slope of the negative diagonal BD.

Property 4. The positive diagonal AC and negative diagonal BD are perpendicular to each other.

Property 5. The angles $\angle \mathrm{ABD}$ and $\angle \mathrm{ACD}$ equal to a common amount $\theta_{1}$ whose range is $\left[0,90^{\circ}\right]$.

Property 6. The diagonals $\mathrm{AC}$ and $\mathrm{BD}$ are equal to a common length $\mathfrak{R}_{1}$ which is just the tile area ABCD, implying that the tile are increases at the same rate as their diagonals.
Property 7. Consequently, note that the angle $\angle \mathrm{ABD}$ (or its equivalent angle $\angle \mathrm{ACD}$ ) is:

$$
\theta_{1}=\tan ^{-1}\left(\mathrm{~m}_{1}\right)
$$

When, $\mathrm{m}_{1}=1$, the angle. When, the angle $\theta_{1}>45^{\circ}$. When $\mathrm{m}_{1}>1, \theta_{1}<45^{\circ}$. The baseline value for the angle is $45^{\circ}$. Another implication is that the angle decreases to zero when the specificity decreases to zero (or equivalently, the points $\mathrm{A}$ and $\mathrm{D}$ collide while the points $\mathrm{B}$ and $\mathrm{C}$ collide together). On the contrary, the angle increases to $90^{\circ}$ when the sensitivity decreases to zero (or equivalently, the points $\mathrm{A}$ and $\mathrm{B}$ collide while the points $\mathrm{C}$ and $\mathrm{D}$ collide together).

All above discussions about the test results pertain to a restrictive scenario of known state about the illness. With no given knowledge about this scenario, the above properties are quite meaningless and obsolete.

Likewise, a complementary mosaic masonry and its tile areas in a unit square as in Fig. 2 below can be constructed to denote the harmonies, $\mathrm{H}_{\mathrm{D}+}=\mathrm{p}_{+} \mathrm{PPV}, \mathrm{H}_{\overline{\mathrm{D}}-}=\mathrm{p}_{-} \mathrm{NPV}$ and discards $\mathrm{H}_{\overline{\mathrm{D}}+}=\mathrm{p}_{+} \mathrm{NPV} \mathrm{H}_{\mathrm{D}-}=\mathrm{p}_{-} \mathrm{PPV}$ with the properties 8 through 14 below.

Property 8. The tile area ABCD in Fig. 2 is $\mathfrak{R}_{2}=$ PPV* NPV which signifies superiority of a diagnostic test about the disease status from a conditional point of view of given diagnostic results. The domain for $\mathfrak{R}_{2}$ is $[0,1]$. Unless both PPV and NPV are large, the tile area, is not large and hence, it is better index than the Shanmugam's index, J.

Property 9. The odds for a diagnostic test to be superior is $\operatorname{odd}_{2}=\left(\Re_{2}^{-1}-1\right)^{-1}$. This odds increases when the tile area $\mathrm{ABCD}$ in Fig. 2 increases.

Property 10. The slope of the positive diagonal AC in Fig. $2 \mathrm{~m}_{2}=\frac{\mathfrak{R}_{2}}{\mathrm{PPV}^{2}}=\frac{\mathrm{NPV}^{2}}{\mathfrak{R}_{2}}$ is which is just minus of the slope of the negative diagonal BD.

Property 11. The positive diagonal $\mathrm{AC}$ and negative diagonal BD are perpendicular to each other.

Property 12. The angles $\angle \mathrm{ABD}$ and $\angle \mathrm{ACD}$ are equal to a common amount $\theta_{2}$ whose range is $\left[0,90^{\circ}\right]$

Property 13. The diagonals $\mathrm{AC}$ and $\mathrm{BD}$ are equal to a common length $\mathfrak{R}_{2}$ which is just the tile area ABCD, implying that the tile are increases at the same rate as their diagonals. 
Property 14. Consequently, note that the angle $\angle \mathrm{ABD}$ (or its equivalent angle $\angle \mathrm{ACD}$ is:

$$
\theta_{2}=\tan ^{-1}\left(m_{2}\right)
$$

When, $\mathrm{m}_{2}=1$ note that $\theta_{2}=45^{\circ}$. When $\mathrm{m}_{2}>1$, the angle becomes $\theta_{2}>45^{\circ}$. When $\mathrm{m}_{2}<1$, the angle is $\theta_{2}<45^{\circ}$. The baseline value for the angle is $45^{\circ}$. Another implication is that the angle decreases to zero when the PPV decreases to zero (or equivalently, the points A and $\mathrm{D}$ collide while the points $\mathrm{B}$ and $\mathrm{C}$ collide together). On the contrary, the angle increases to $90^{\circ}$ when the NPV decreases to zero (or equivalently, the points $\mathrm{A}$ and $\mathrm{B}$ collide while the points $\mathrm{C}$ and $\mathrm{D}$ collide together).

These discussions about the illness pertain to a restrictive scenario of given diagnostic test result. With no given knowledge about this scenario, above properties are quite meaningless and obsolete. The discussions based on Fig. 1 and 2 are one sided but they are quite parallel. They need to be crossbred to be unconditional and realistic. Such crossbreeding is feasible by superimposing both mosaics masonries on one another, as seen in Fig. 3. In the crossbred results, subscripts are dropped.

A dependence measure, $\mathrm{C}$ of the event, $\mathrm{D}$ of being with illness and the event, + of getting positive result in a diagnostic test is defined and identified. The area $\mathrm{C}$ is zero when the events $\mathrm{D}$ and + are independent.

With PPV $=$ NPV or $S_{e}=S_{p}$ or when the tiles do not overlap as an extreme case, the dependence measure $\mathrm{C}$ is zero. When the two tiles perfectly overlap as another extreme case, the dependence measure $\mathrm{C}$ is one. Otherwise, there is an intermediate dependency level between the illness and diagnostic test outcomes. The measure $\mathrm{C}$ is in the closed interval $[0,1]$. To further visualize and understand its implications, the rectangular segment enclosing $\mathrm{C}$ is zoomed out as displayed in Fig. 4. Subsequently, several properties of the dependence measure $\mathrm{C}$ can be extracted using fundamental laws of triangles and rectangles as they are done below. These properties help to develop a new assessment technique to check superiority of a diagnostic test. There are advantages in this new visual method of assessment as it integrates the masonries in Fig. 1 and 2.

Property 15. The area of the rectangle ABCD in Fig. 4 is Equation 5:

$$
\begin{aligned}
& \Re=\left(\mathrm{S}_{\mathrm{p}}+\mathrm{PPV}-1\right)\left(\mathrm{S}_{\mathrm{e}}+\mathrm{NPV}-1\right) \\
& =\left(\mathrm{R}_{1}-\mathrm{I}\right)+\left(\mathrm{R}_{2}-\mathrm{J}\right) \\
& +\left(\mathrm{S}_{\mathrm{p}} \mathrm{NPV}+\mathrm{S}_{\mathrm{e}} \mathrm{PPV}-1\right)
\end{aligned}
$$

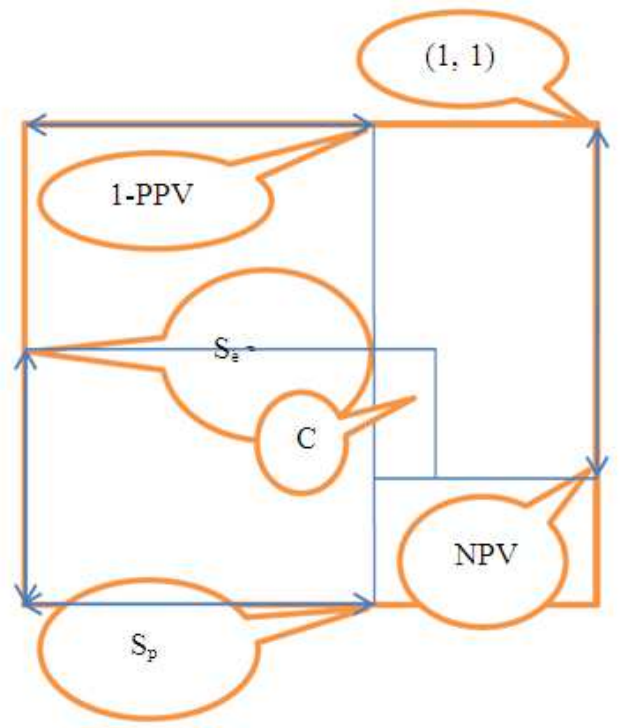

Fig. 3. Dependence measure, $\mathrm{C}$

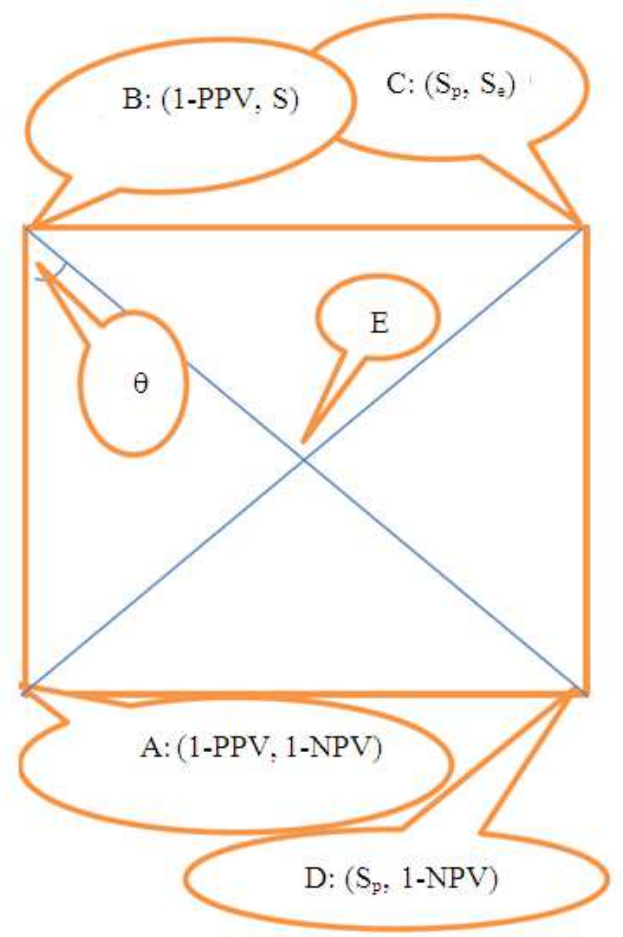

Fig. 4. Dependence measure, $C$ when $0<\theta<90^{\circ}$

Which signifies crossbred measure about superiority of a diagnostic test. The domain for $\mathfrak{R}$ is $[0,1]$. Unless all (which includes PPV, NPV, sensitivity and 
specificity) are large, the tile area, $\mathfrak{R}$ is not large. Hence, the tile area, $\mathfrak{R}$ is better than the Youden index, I or Shanmugam's index, J.

Property 16. The odds for a diagnostic test to be superior is Equation 6:

$$
\begin{aligned}
& \text { odd }=\left(\Re^{-1}-1\right)^{-1} \\
& =\left[\left(\mathrm{S}_{\mathrm{p}}+\mathrm{PPV}-1\right)^{-1}\left(\mathrm{~S}_{\mathrm{e}}+\mathrm{NPV}-1\right)^{-1}-1\right]^{-1}
\end{aligned}
$$

The odds increases when the area $\mathfrak{R}$ increases.

Property 17. Note that the intersection point E of the diagonals in Fig. 4 has co-ordinates $\mathrm{E}:\left(\frac{\mathrm{S}_{\mathrm{e}}}{\mathrm{m}}, 1+\mathrm{S}_{\mathrm{e}}-\mathrm{NPV}\right)$ where Equation 7 :

$\mathrm{m}=\frac{\mathrm{S}_{\mathrm{e}}+\mathrm{NPV}-1}{\mathrm{~S}_{\mathrm{p}}+\mathrm{PPV}-1}$

$=\frac{\left(\mathrm{S}_{\mathrm{e}}+\mathrm{NPV}-1\right)^{2}}{\mathfrak{R}}$

$$
=\frac{\Re}{\left(\mathrm{S}_{\mathrm{p}}+\mathrm{PPV}-1\right)^{2}}
$$

Is the slope of the inclining diagonal $\mathrm{AC}$ and minus slope of the declining diagonal BD.

Property 18. The length of the positive diagonal AC and the length of the negative diagonal $\mathrm{BD}$ are equal to a common amount Equation 8:

$\Re^{2}=\left(\mathrm{S}_{\mathrm{p}}+\mathrm{PPV}-1\right)^{2}+\left(\mathrm{S}_{\mathrm{e}}+\mathrm{NPV}-1\right)^{2}$

Property 19. The equation of the inclining diagonal, $\mathrm{AC}$ is:

$$
\mathrm{y}=\mathrm{S}_{\mathrm{e}}+\mathrm{m}\left(\mathrm{x}-\mathrm{S}_{\mathrm{p}}\right)
$$

Or equivalently Equation 9:

$\mathrm{y}=1-\mathrm{NPV}+\mathrm{m}(\mathrm{x}+\mathrm{PPV}-1)$

where, $\mathrm{m}$ is the slope of the diagonal AC.

Property 20. The equation of the declining diagonal, BD is Equation 10:

$\mathrm{y}=\mathrm{S}_{\mathrm{e}}-\mathrm{m}(\mathrm{x}+\mathrm{PPV}-1)$

Or equivalently Equation 11:

$y=1-N P V-m\left(x-S_{p}\right)$

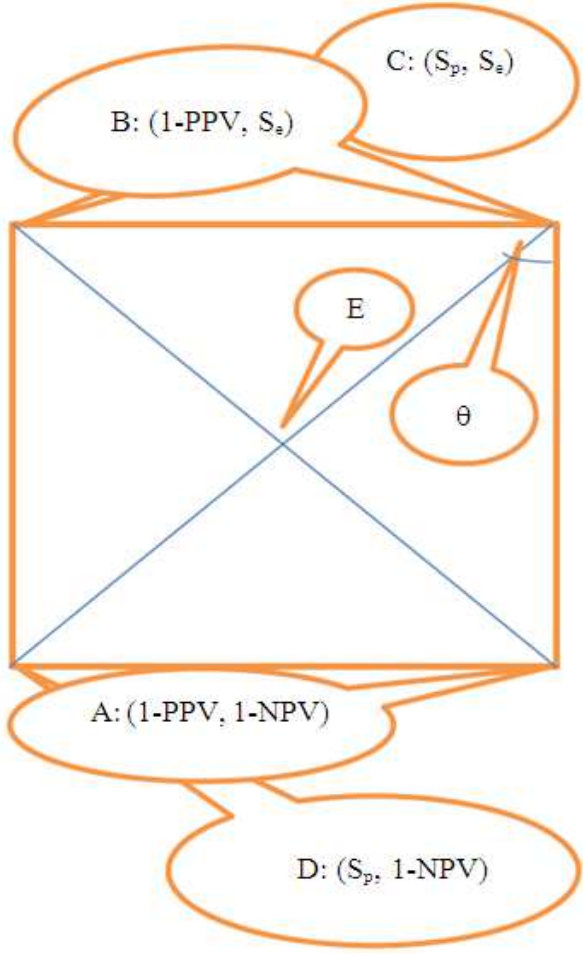

Fig. 5. Dependence measure, $C$ when $-90^{\circ}<\theta<0$

Property 21. The triangles $\triangle \mathrm{ABD}$ and $\triangle \mathrm{ACD}$ are congruent and consequently, the angles. $\angle \mathrm{ABD}=\theta=$ $\angle \mathrm{ACD}$.

Property 22. Furthermore, note that Equation 12:

$\theta=\tan ^{-1}\left(\frac{1}{m}\right)=\tan ^{-1}\left(\frac{S_{p}+P P V-1}{S+N P V-1}\right)$

The range for the angle $\theta$ in (12) is $\left[-90^{\circ}, 90^{\circ}\right]$. When, the corners and diagonals of Fig. 4 are flipped to appear like in Fig. 5 but hold the above mentioned properties of Fig. 4.

Property 23. The optimal angle is $\theta= \pm 45^{\circ}$ and it occurs when $S_{p}+P P V=S_{e}+N P V$. As an extreme, when $\theta \rightarrow 0$, the points $\mathrm{A}$ and $\mathrm{D}$ collide as points $\mathrm{B}$ and $\mathrm{C}$ collide. Likewise, as an extreme, when $\theta \rightarrow 90^{\circ}$, the points $\mathrm{B}$ and A collide as points $\mathrm{C}$ and $\mathrm{D}$ collide.

The measure $\mathrm{m}$ is a better index than $\mathrm{I}$ and $\mathrm{J}$ to check whether a diagnostic test is superior. The range for $\mathrm{m}$ is an unbounded bracket $(-\infty, \infty)$. Hence, in fact, more preferable index is the bounded angle $\theta$, compared to unbounded measure $\mathrm{m}$. 
Now, the article focuses on developing a technique to obtain the significance level of an estimated angle $\theta$ in (12). For this purpose, the estimated angle $\theta$ in (12) is assumed to follow a uniform distribution $\cup\left(-90^{\circ}, 0^{\circ}\right)$ when $\mathrm{m}$ in (12) is negative and a uniform distribution $\cup$ $\left(0^{\circ}, 90^{\circ}\right)$ when $\mathrm{m}$ in $(12)$ is positive. When $\mathrm{m}$ in (12) is positive, the expected value is $\mathrm{E}(\theta)=45^{\circ}$ When $\mathrm{m}$ in (12) is negative, the expected value is $\mathrm{E}(\theta)=45^{\circ}$.

In both cases, the dispersion $\sigma_{\theta}^{2}$ is same. Is an estimated angle $\theta$ in (12) too small or too large compared to its expected value? The answer requires performing a hypothesis test using normal distribution as an approximation for the estimated angle $\theta$ in (12). For this purpose, we use $6 \sigma \approx\left(90^{\circ}-0^{\circ}\right)$. That means $\sigma \approx 15^{\circ}$ the null E hypothesis $\mathrm{H}_{0}:|\theta|-|\mathrm{E}(\theta)|=0$ means the estimated angle $\theta$ in (12) is near its expected value. The alternative hypothesis $\mathrm{H}_{\mathrm{a}}:|\theta|-|| \mathrm{E}(\theta) \mid \neq 0$ means the estimated angle $\theta$ in (12) is significantly small or large. How likely is the null hypothesis to be false and it is indicated by its p-value Equation 13:

$\mathrm{p}-$ value $\approx 2 \operatorname{Pr}\left(\mathrm{Z}>\left|\frac{\left|\tan ^{-1}\left(\frac{1}{\mathrm{~m}}\right)\right|-45^{\circ}}{15}\right|\right)$

The null hypothesis is rejected when the $p$-value is smaller. With a selected level of significance a, the statistical power to reject $\mathrm{H}_{\mathrm{o}}|\theta|-|\mathrm{E}(\theta)|=0$ in favor of a true specific alternative hypothes $\mathrm{H}_{\mathrm{a}}: \theta=\left|\theta_{1}\right|$ is power Equation 14:

$$
\begin{aligned}
& \approx \operatorname{Pr}\left[-\mathrm{z}_{\mathrm{a} / 2}\right]\left|\frac{\left|\tan ^{-1}\left(\frac{1}{\mathrm{~m}}\right)\right|-\theta_{1}^{\circ}}{\left|\tan ^{-1}\left(\frac{1}{\mathrm{~m}}\right)\right|-45^{\circ}}\right|<\mathrm{Z} \\
& \left.<\mathrm{z}_{\mathrm{a} / 2}\left|\frac{\left|\tan ^{-1}\left(\frac{1}{\mathrm{~m}}\right)\right|-\theta_{1}^{\circ}}{\left|\tan ^{-1}\left(\frac{1}{\mathrm{~m}}\right)\right|-45^{\circ}}\right|\right]
\end{aligned}
$$

To illustrate, $\theta_{1}^{\circ}=0^{\circ}, 15^{\circ}, 30^{\circ}, 60^{\circ}, 75^{\circ}$ and $90^{\circ}$ withare considered later in the article. The above concepts and tools are illustrated using medical and health data sets from the literature.

\subsection{Illustration with Medical Data}

We now examine how effective our visual methodology of section 2 works in several medical data in Table 1-5.
Consider the data about the presence or absence of rotavirus among a random sample of $n=393$ children who vomited in Table 1 as reported in Tabue (1986).

Consider the mammogram data of a random sample of $\mathrm{n}=3,000$ women from page 21 in Zhou et al. (2002) as in Table 2 below.

Whether infection occurred after surgery in a random sample of $\mathrm{n}=39$ men in a hospital at Austin, Texas while not all of them had gone through MRI. Their results are reproduced from Shanmugam (2008) in Table 3.

Two nurses independently examined tympanic membrane in the eardrum of a random sample of $n=100$ ear patients about their ear infection. The results are displayed in Table 4 as reported in Le (2003).

Whether surgery has helped to control cancer cells was answered by a random sample of $n=41$ insured cases is displayed in Table 5 as reported in Agresti (2007).

The slope $\mathrm{m}$, angle $\theta, \mathrm{p}$-value (that is, the probability tor null hypothesis $\left.\mathrm{H}_{\mathrm{o}}:|\theta|-|\mathrm{E}(\theta)|=0\right)$ to be true and statistical power (that is, the probability to accept a specific true alternative hypothesis $\mathrm{H}_{1}: \theta_{1}=30^{\circ}$ for the data in Table 1 through 5 are summarized in Table 6. In all the data sets, except the first data on rotavirus, the null hypothesis $H_{o}:|\theta|-|| E(\theta) \mid=0$ is rejected and it means that the estimated angle $\theta$ is insignificant compared to its expected value, $45^{\circ}$. In an event $\theta_{1}=30^{\circ}$, the chance of accepting it is excellent in the $1 \mathrm{st}$, $3 \mathrm{rd}$ and 5 th but reasonable in other data sets. According to indices I or J, the diagnostic test was slightly superior when the illness is rota virus, mammogram, surgical infection and tympanic membrane but not in surgery to control cancer. The slope $\mathrm{m}$ is more in the case of tympanic membrane and small in the case of surgery to control cancer. The angle $\theta$ is above its expected angle $45^{\circ}$ in the case of "does surgery control cancer?" but below in the case of rotavirus, mammogram, surgical infection and tympanic membrane.

Note that when $\delta=|\theta|-\left|\theta_{1}\right|$ is near zero, it is called contiguity in the literature. The power is low in the contiguity locations. The contiguity occurs, with $\theta_{1}=30^{\circ}$ and the estimated $\theta$ (Table 6), in the case of mammogram and tympanic membrane data sets and hence, the power curve is low in those contiguity locations (Fig. 6).

\subsection{Illustration with Health Data}

We now examine how superior our visual methodology of section 2 works in several health data in Table 7-13. 
Table 1. Rotavirus among children

\begin{tabular}{lccl}
\hline Rotavirus? $\rightarrow$ Vomit? & Yes (D) & No $\bar{D}$ & Sum \\
\hline Yes $(+)$ & 146 & 130 & 276 \\
No (-) & 22 & 95 & 117 \\
Sum & 168 & 225 & 393 \\
\hline
\end{tabular}

Table 2. Mammogram among women

\begin{tabular}{lcll}
\hline Cancer? $\rightarrow$ mammogram? & Yes (D) & No D & Sum \\
\hline Yes $(+)$ & 29 & 1881 & 1910 \\
No $(-)$ & 1 & 1089 & 1090 \\
Sum & 30 & 2970 & 3000
\end{tabular}

Table 3. Patients surgical infection

\begin{tabular}{lccr}
\hline Infection? $\rightarrow$ MRI? & Yes (D) & No $\overline{\mathrm{D}}$ & Sum \\
\hline Yes (+) & 20 & 4 & 24 \\
No (-) & 4 & 11 & 15 \\
Sum & 24 & 15 & 39 \\
\hline
\end{tabular}

Table 4. Tympanic membrane infection

\begin{tabular}{lccc}
\hline Nurse1? $\rightarrow$ nurse2? & Yes (D) & No $\overline{\mathrm{D}}$ & Sum \\
\hline Yes (+) & 35 & 20 & 55 \\
No (-) & 10 & 35 & 45 \\
Sum & 45 & 55 & 100 \\
\hline
\end{tabular}

Table 5. Surgery to control cancer

\begin{tabular}{llll}
\hline Cancer controlled surgery? $\rightarrow$ & Yes $(\mathrm{D})$ & No $\overline{\mathrm{D}}$ & Sum \\
\hline Yes $(+)$ & 2 & 21 & 23 \\
No $(-)$ & 3 & 15 & 18 \\
Sum & 5 & 36 & 41 \\
\hline
\end{tabular}

Table 6. Assessment summary for data in Table 1 through 6

\begin{tabular}{lrrcc}
\hline Data type & \multicolumn{1}{c}{$\mathrm{m}$} & \multicolumn{1}{c}{$\theta^{0}$} & $\mathrm{p}$-value & Power \\
\hline Rota virus & -14.00 & -4.1 & 0.01 & 0.78 \\
Mammogram & -1.56 & -33.0 & 0.41 & 0.32 \\
Surgical infection & 1.00 & 45.0 & 1.00 & 0.99 \\
Tympanic membrane & 2.04 & 26.0 & 0.21 & 0.31 \\
$\begin{array}{l}\text { Does surgery control } \\
\text { cancer? }\end{array}$ & -0.50 & -64.0 & 0.19 & 0.98 \\
\hline
\end{tabular}

Table 7. Depression with anxiety

\begin{tabular}{lclr}
\hline Depression anxiety & Yes (D) & No $\bar{D}$ & sum \\
\hline Yes (+) & 202 & 34 & 236 \\
No (-) & 28 & 13 & 41 \\
Sum & 230 & 47 & 277 \\
\hline
\end{tabular}

Table 8. Small-pox incidence versus vaccinated children

\begin{tabular}{lclc}
\hline Smallpox? $\rightarrow$ vaccinated? & Yes (D) & No $\bar{D}$ & Sum \\
\hline Yes $(+)$ & 4 & 119 & 123 \\
No (-) & 11 & 128 & 139 \\
Sum & 15 & 147 & 262 \\
\hline
\end{tabular}

Table 9. Who used condom

\begin{tabular}{llll}
\hline Husband $\rightarrow$ wife? & Yes $(\mathrm{D})$ & No $\overline{\mathrm{D}}$ & Sum \\
\hline Yes $(+)$ & 45 & 6 & 51 \\
No $(-)$ & 7 & 40 & 47 \\
Sum & 52 & 46 & 98 \\
\hline
\end{tabular}

Table 10. X-ray of patients

\begin{tabular}{lcrc}
\hline Tuberculosis? $\rightarrow$ x-ray? & Yes (D) & No $\bar{D}$ & Sum \\
\hline Yes $(+)$ & 22 & 51 & 73 \\
No $(-)$ & 8 & 1739 & 1747 \\
Sum & 30 & 1790 & 1820 \\
\hline
\end{tabular}

Table 11. Vietnam Veteran's health

\begin{tabular}{lcrc}
\hline Vietnamveteran? $\rightarrow$ nightmare? & Yes (D) & No $\bar{D}$ & Sum \\
\hline Yes (+) & 197 & 85 & 282 \\
No (-) & 577 & 925 & 1502 \\
Sum & 774 & 1010 & 1784 \\
\hline
\end{tabular}

Table 12. Screening for diabetes

\begin{tabular}{llcc}
\hline diabetic? $\rightarrow$ more sugar? & Yes (D) & No $\bar{D}$ & Sum \\
\hline Yes (+) & 56 & 49 & 105 \\
No (-) & 14 & 461 & 475 \\
Sum & 70 & 510 & 580 \\
\hline
\end{tabular}

Table 13. HIV among homeless

\begin{tabular}{lccl}
\hline Used intravenous? $\rightarrow$ HIV? & Yes (D) & No $\bar{D}$ & Sum \\
\hline Yes (+) & 7 & 4 & 11 \\
No (-) & 4 & 10 & 14 \\
Sum & 11 & 14 & 25 \\
\hline
\end{tabular}

Table 14. Assessment summary for data in Tables 13 through 13

\begin{tabular}{lrrcc}
\hline Data type & $\mathrm{m}$ & $\mathrm{q}$ & $\mathrm{p}$-value & Power \\
\hline Depression and anxiety & 1.47 & 34 & 0.47 & 0.54 \\
Small-pox and vaccination & -1.90 & -27 & 0.24 & 0.23 \\
Condom use by couples & 0.95 & 46 & 0.93 & 0.99 \\
TB and X-ray & 2.67 & 21 & 0.10 & 0.55 \\
Veteran and nightmare & -0.20 & -78 & 0.02 & 0.99 \\
Diabetic and more sugar & 1.76 & 30 & 0.30 & 0.04 \\
HIV and homeless & 1.00 & 45 & 1.00 & 0.99 \\
\hline
\end{tabular}

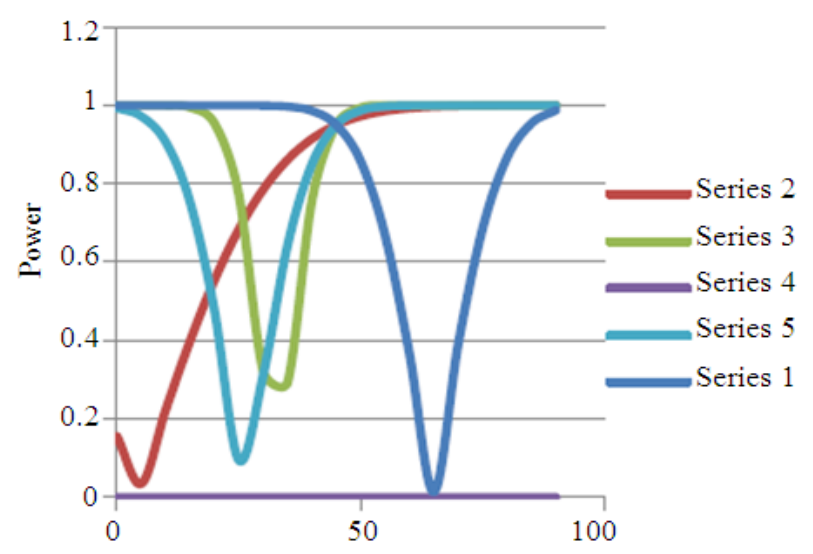

Fig. 6. Power curves for data sets (series 1, 2, 3, 4 and 5 are for rotavirus, mammogram, surgical infection, tympanic membran and surgery control cancer respectively) 


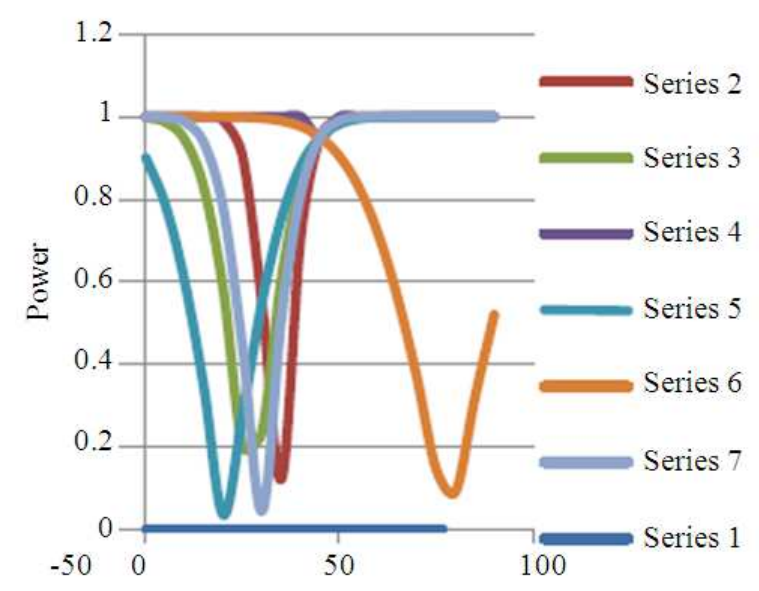

Fig. 7. Power curves for data sets (series 1, 2, 3, 4, 5 and 6 are for depression-anxiety, smallpox-vaccination, condom use by couples, TB-x ray, Vietnam veteran's nightmare, diabetic-more sugar and HIV-homeless respectively)

A random sample of $n=277$ patients in a mental hospital were asked about their depression and/or anxiety. The results are reproduced in Table 7 from Altham and Colton (2005).

Tabue (1986) collected data on smallpox incidence among children and they are displayed in Table 8.

Ninety-eight randomly chosen heterosexual couples mentioned that at least one of them had HIV infection. They answered a question whether one or both used condom as in Table 9, according to Le (2003).

A random sample of $\mathrm{n}=1820$ individuals was asked about whether they had x-ray and tuberculosis infection. Their responses are displayed in Table $\mathbf{1 0}$ as mentioned in Le (2003).

How many had nightmare among the veterans who served in Vietnam war are reproduced in Table $\mathbf{1 1}$ from Le (2003).

In an article about the efficacy of screening tests, Remsin and Wilkerson (1961) reported the data in Table 12. Randomly sampled twenty-five cases are classified in Table 13 as reported in Schork and Remington (2000).

The Youden index I, Shanmugam's index J, slope $\mathrm{m}$ and the angle $\theta$ for the data in Table 7-13 are summarized in Table 14. According to indices I and J, the diagnostic test was superior when the illness is depression-anxiety, smallpox-vaccination, condom use by couples, TB-x ray, Vietnam veteran nightmare, diabetic-more sugar and HIV among homeless data sets. The slope $m$ is and the estimated angle $\theta$ are displayed in Table 14. The estimated angles are around its expected value $45^{\circ}$ but are not too small.
The probability tor the null hypothesis $\mathrm{H}_{0}:|\theta|-|\mathrm{E}(\theta)|=$ 0 to be true and the statistical power (that is, the probability to accept a specific true alternative hypothesis $\mathrm{H}_{1}: \theta_{1}=30^{\circ}$ for the data in Table 7 through 13 are summarized in Table 14. In all the data sets, the null hypothesis $\mathrm{H}_{0}:|\theta|-|\mathrm{E}(\theta)|=0$ is rejected only in the Vietnam veteran's data but not in other data sets based on their $p$-value. It means that the estimated angle $\theta$ is significantly different from its expected value, $45^{\circ}$ only in veterans data but not in other data sets In an event $\theta_{1}=$ $30^{\circ}$, the chance of accepting it is excellent or reasonable in all data sets except in "diabetic and more sugar" data set. A reason for low power is it's near zero contiguity. When $\delta=|\theta|-\left|\theta_{1}\right|$ is near zero, it is called contiguity in the literature. The power is low in the contiguity locations. The contiguity occurs, with $\theta_{1}=30$ and the estimated $\theta$ (Table 14), in the case of diabetic and more sugar data set and hence, the power curve is low in those contiguity locations (Fig. 7).

\section{CONCLUSION}

Based on the complementary mosaic masonries which are introduced in this article, it is quite simple and easy to visualize how superior is a diagnostic test without conditioning on test results or on the presence or absence of disease status. Applied researchers might be curious to trace factors which cause a diagnostic test to be superior or inferior. An answer to their curiosity depends on a suitable regression methodology and it is necessary. Developing a suitable regression methodology is a topic for future research. Additional data are needed to check such regression methodology.

\section{REFERENCES}

Agresti, A., 2007. An Introduction to Categorical Data Analysis. 2nd Edn., Wiley-Interscience, Hoboken, ISBN-10: 0471226181, pp: 352.

Altham, P. and T. Colton, 2005. Encyclopedia of Biostatistics. 2nd Edn., John Wiley, Chichester, ISBN-10: 047084907X, pp: 6100.

Le, C.T., 2003. Introductory Biostatistics. 1st Edn., John Wiley and Sons, Hoboken, ISBN-10: 9780471458562, pp: 552.

Remsin, Q.R. and H.C. Wilkerson, 1961. The efficiency of screening tests for diabetes. J. Chronic Dis., 13: 6-21. DOI: 10.1016/0021-9681(61)90041-8 
Schork, M.A. and R.D. Remington, 2000. Statistics with Applications to the Biological and Health Sciences. 3rd Edn., Prentice Hall, Englewood, ISBN-10: 0130223271, pp: 478.

Shanmugam, R., 2008. Double anchored syllogism for medical scenarios. J. Stat. Appli., 3: 186-206.

Tabue, A., 1986. Sensitivity, specificity and predictive values: A graphical approach. Stat. Med., 5: 585561. DOI: $10.1002 / \mathrm{sim} .4780050606$
Youden, W.J., 1950. Index for rating diagnostic tests. Cancer, 3: 32-35. PMID: 15405679

Zhou, X.H., N.A. Obuchowski and D.K. McClish, 2002. Statistical Methods in Diagnostic Medicine. 1st Edn., Wiley-Interscience, New York, ISBN-10: 9780470317082, pp: 464. 